\title{
PROMOCIÓN DE LA SALUD ORAL Y PERSPECTIVAS PARA EL 2020 DE LA REVISTA PERUANA DE MEDICINA EXPERIMENTAL Y SALUD PÚBLICA
}

\author{
PROMOTION OF ORAL HEALTH AND PROSPECTS FOR 2020 FOR THE REVISTA \\ PERUANA DE MEDICINA EXPERIMENTAL Y SALUD PUBLICA
}

\author{
Akram Hernández-Vásquez ${ }^{1, a}$, Zuño Burstein ${ }^{1, b}$
}

Las enfermedades de la cavidad bucal (patología oral) representan uno de los más grandes desafíos para la salud global debido a su alta prevalencia, sus vínculos con la salud general y la gran carga económica que generan ${ }^{(1)}$. Este asunto, merece la atención de los gobiernos, tomadores de decisiones e investigadores, por ser un problema actual y creciente de salud pública, y que requiere una serie de medidas costo-efectivas en prevención y promoción. La Revista Peruana de Medicina Experimental y Salud Pública (RPMESP) cumpliendo con su función como órgano oficial de difusión científica del Instituto Nacional de Salud (INS) presenta en este número el tema «Higiene y Salud Oral», que difunde una serie de contribuciones sobre la temática y propone oportunidades de mejora dentro de la salud pública odontológica, a nivel regional y local.

En la sección de artículos originales, se presenta un estudio de Azañedo et al. sobre el uso de servicios de salud oral en adultos mayores peruanos a partir de la Encuesta Demográfica y de Salud Familiar, los autores reportan que sólo tres de cada diez adultos mayores usaron un servicio odontológico en los seis meses previos a la encuesta y que existen factores sociodemográficos asociados a un mayor uso de servicios de salud oral, como el nivel educativo superior, la tenencia de un seguro de salud y el mayor nivel de riqueza ${ }^{(2)}$. Por lo descrito, las políticas y programas en salud pública dirigidas hacia esta población deben tomar en cuenta estas características para mejorar el acceso a los servicios odontológicos.

Es de resaltar que el cepillado dental con pastas dentales fluoradas están catalogadas como una de las medidas y estrategias más efectivas en la salud pública dental. Al respecto, el estudio de Solis et al. titulado «Tendencia y factores asociados a la frecuencia de cepillado dental en menores de doce años, Perú 2013-2018» expone una mejora constante en cuanto a la frecuencia de cepillado adecuado (dos veces al día como mínimo) de $79,0 \%$ en 2013 a $83,9 \%$ en 2018 , pero a la vez, nos muestra una serie de brechas con una menor frecuencia de cepillado en el área rural y en la sierra peruana ${ }^{(3)}$.

Además, dentro de los artículos originales breves, se presenta el artículo «Cepillado dental y niveles de flúor en pastas dentales usadas por niños peruanos menores de 12 años» ${ }^{(4)}$, con importantes hallazgos en materia de prevención y regulación del nivel de flúor empleado en la fabricación de pastas dentales en nuestro país, basado en que la evidencia y la guía de Ministerio de Salud establecen un nivel de flúor en pastas dentales mayor a 1000 ppm para lograr efectos preventivos sobre la caries dental. El estudio reportó que un $7,8 \%$ de los evaluados no se cepillaba los dientes y que un buen número de niños peruanos aún siguen utilizando pastas dentales sin efecto preventivo.

Por otro lado, el estudio «Condiciones de salud oral, higiene oral y estado nutricional en niños que acuden a un establecimiento de salud de la región Huánuco, Perú» ${ }^{(5)}$, nos muestra los resultados de una investigación en niños de una de las regiones más pobres de nuestro país y con mayor prevalencia de desnutrición, el estudio reporta la existencia de una relación entre las condiciones de salud oral e higiene oral deficientes y un inadecuado estado nutricional.

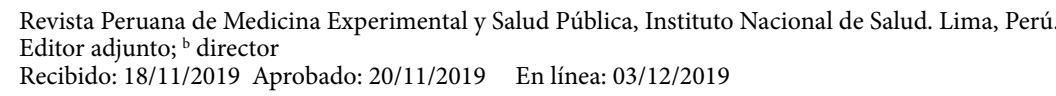

Citar como: Hernández-Vásquez A, Burstein Z. Promoción de la salud oral y perspectivas para el 2020 de la Revista Peruana de Medicina Experimental y Salud Pública. Rev Peru Med Exp Salud Publica. 2019;36(4):551-2. doi: 10.17843/rpmesp.2019.364.4982. 
Otro de los problemas vigentes que afecta la salud oral de nuestro país es la migración, lo que puede ocasionar en esta población la presencia de necesidades aún no cubiertas por los sistemas de salud, que podrían verse exacerbados por una mayor demanda de atención, y actuar como un determinante social con impacto en los indicadores de salud oral. En ese contexto, el presente número incluye el simposio «La migración sur/sur como un determinante social de impacto en las desigualdades e inequidades en salud bucal en Latinoamérica» (6), que señala la necesidad de generación de evidencias basadas en la realidad social de los migrantes y de los países hacia los cuales migran, proponiendo un marco conceptual que permita estudiar este fenómeno social y su relación con la salud bucal desde diferentes enfoques metodológicos en el contexto latinoamericano.

Así, la RPMESP cierra un nuevo año puesta al servicio de la salud pública en especial de nuestro país y de la región latinoamericana, permitiendo la difusión de artículos de alta calidad y velando por el cumplimiento de las directrices éticas en investigación y publicación biomédica. Lo anterior no sería posible sin el compromiso del equipo editorial y comité editor, y de las contribuciones de autores y revisores.

Hasta el 17 de noviembre de 2019, se ha recibido el mayor número de artículos $(n=581)$ en comparación con los años anteriores $(n=526$ en 2018, n=507 en 2017) a través de nuestra plataforma Open Journal System. Este incremento constante es motivo de satisfacción para el comité editor de la RPMESP y puede atribuirse a la calidad y a la difusión oportuna que tiene la revista. Sin embargo, quedan muchas metas por lograr, como la publicación en idioma inglés de la totalidad de sus artículos, lo que permitiría una mayor visualización a nivel mundial. En tal sentido, la RPMESP traducirá al inglés parte de los artículos de cada número, lo cual, estamos seguros, atraerá un mayor número de manuscritos e incrementará el impacto de la revista.
Reconociendo la necesidad de una mejora constante en sus procesos, diversidad de temas, calidad de los manuscritos recibidos y motivos de no aceptación [7], la RPMESP considera que es importante actualizar sus instrucciones para autores. Hacemos énfasis que en la sección especial recibimos contribuciones con enfoques metodológicos, procedimientos o instrumentos nuevos o alternativos que puedan ser empleados en investigaciones en el campo de la salud pública o medicina experimental. Asimismo, se han definido las formas más frecuentes de faltas éticas en publicación (plagio, autoría inadecuada y publicación redundante) para conocimiento de los autores, reafirmando nuestra adherencia a las recomendaciones del Committee on Publication Ethics (COPE), la World Association of Medical Editors (WAME), el International Committee of Medical Journal Editors (ICMJE) y el Council of Science Editors en nuestros procesos editoriales. Por último, se precisa la forma de evaluación y declaración de los potenciales conflictos de interés para los manuscritos, que incluyen entre sus autores a miembros de nuestro comité editor.

Para el 2020 se ha priorizado el desarrollo de cuatro temas actuales y relevantes para la salud pública. El primer número abordará el tema de "Género y salud» con fecha de recepción de artículos hasta el 14 de enero de 2020 . El segundo número está dedicado a la «Salud urbana» con recepción de artículos hasta el 22 de abril de 2020. Para el tercer número se desarrollará el tema de «Medicina del viajero» con cierre de recepción de artículos para el 22 de julio de 2020. Y por último, el cuarto número será sobre «Innovaciones en Salud Pública» y la recepción de artículos será hasta el 21 de octubre de 2020.

Desde la RPMESP hacemos la invitación a la comunidad científica y académica para que sigan enviando sus contribuciones en el campo de la salud pública y medicina experimental, los cuales deberán cumplir los requisitos exigidos en nuestras nuevas instrucciones para autores.

\section{REFERENCIAS BIBLIOGRÁFICAS}

1. Kassebaum NJ, Smith AGC, Bernabe E, Fleming TD, Reynolds AE, Vos T, et al. Global, Regional, and National Prevalence, Incidence, and Disability-Adjusted Life YearsforOralConditionsfor 195 Countries, 1990-2015: A Systematic Analysis for the Global Burden of Diseases, Injuries, and Risk Factors. J Dent Res. 2017;96(4):3807.doi: $10.1177 / 0022034517693566$

2. Azañedo D, Vargas-Fernández R, RojasRoque C. Factores asociados al uso de servicios de salud oral en adultos mayores peruanos: análisis secundario de encuesta poblacional, 2018. Rev Peru Med Exp Salud Publica. 2019;36 (4):553-561. doi: 10.17843/rpmesp.2019.364.4724

3. Solis G, Pesaressi E, Mormontoy W. Tendencia y factores asociados a la frecuencia de cepillado dental en menores de doce años, Perú 2013-2018. Rev Peru Med Exp Salud Publica. 2019;36(4):56272. doi: 10.17843/rpmesp.2019.364.4888

4. Hernández-Vásquez A, Azañedo D. Cepillado dental y niveles de flúor en pastas dentales usadas por niños peruanos menores de 12 años. Rev Peru Med Exp Salud Publica. 2019;36(4):646-52. doi: 10.17843/rpmesp.2019.364.4900

5. Vargas-Palomino KE, ChipanaHerquinio CR, Arriola-Guillén LE. Condiciones de salud oral, higiene oral y estado nutricional en niños que acuden a un establecimiento de salud de la región Huánuco, Perú. Rev Peru Med
Exp Salud Publica. 2019;36(4):653-7. doi: 10.17843/rpmesp.2019.364.4891

6. Murillo-Pedrozo AM, Agudelo-Suárez AA. La migración sur/sur como un determinante social de impacto en las desigualdades e inequidades en salud bucal en Latinoamérica. Rev Peru Med Exp Salud Publica. 2019;36(4):692-9. doi: 10.17843/rpmesp.2019.364.4908

7. Arroyo-Hernández $\mathrm{H}$, Huarez $\mathrm{B}$. Tendencia y características de los manuscritos recibidos y rechazados en la Revista Peruana de Medicina Experimental y Salud Pública entre 2011 y 2017. Rev Peru Med Exp Salud Publica. 2019;36(2):28187. doi: 10.17843/rpmesp.2019.362.4190 